$\exists$

\title{
Recycling integrins
}

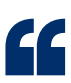

...highlight

a role for

integrin

trafficking in

cell division...
Although it is now well established that vesicular transport has important roles in both cell migration and cytokinesis, the mechanisms by which this occurs remain mostly unclear. Two studies now provide insights into the regulation of integrin trafficking in both processes by the Rab family of small GTPases. Caswell et al. show that Rab-coupling protein (RCP) coordinates endocytic recycling of integrins and promotes tumour cell migration, whereas Pellinen and colleagues highlight a role for integrin trafficking in cell division by showing that RAB21regulated integrin trafficking is essential for cytokinesis.

Based on the analogy between the changes in cell morphology during migration and mitosis, Pellinen and colleagues postulated that RAB21 - which has been shown to regulate integrin trafficking during cell migration - could function to support this process during cell division.

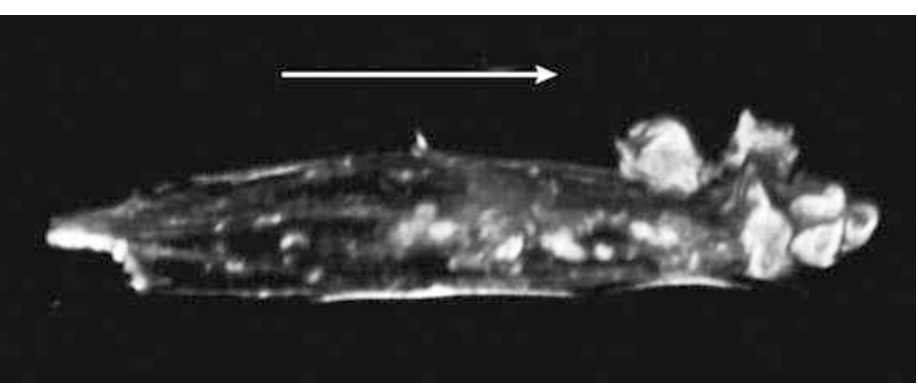

Three-dimensional reconstruction of an ovarian tumour cell migrating in response to osteopontin.Note the presence of numerous actin-rich ruffles extending upward from the cell front. The direction of migration is indicated by the arrow. Image courtesy of Jim Norman, Beatson Institute, Glasgow, United Kingdom.
The authors show that targeted trafficking of integrins to and from the cleavage furrow is regulated by RAB21 and is required for successful cytokinesis. Loss of RAB21 activity, defective integrin-RAB21 association or blocking of integrin endocytosis all result in defective cytokinesis and binuclear cells.

The authors also report a chromosomal deletion in the RAB21 locus in human cancer cells that results in the loss of RAB21 gene expression and leads to the accumulation of multinucleated cells. Re-expression of $R A B 21$ rescues this phenotype. So, RAB21-regulated integrin trafficking is essential for normal cell division, and its defects might contribute to multinucleation and genomic instability, which are hallmarks of cancer.

As RAB11 proteins coordinate integrin recycling during cell migration, Caswell et al. reasoned that there must be at least one RAB11 effector that mediates this process. So, they investigated a potential role for the class I RAB11 family of interacting proteins (FIPs) in coordinating the trafficking of integrins during tumour cell migration in three-dimensional (3D) microenvironments. Blocking the adhesive function of $\alpha v \beta 3$ integrin promoted the association of RCP (a class I RAB11 FIP) with $\alpha 5 \beta 1$ integrin, and drove RCP-dependent recycling of $\alpha 5 \beta 1$ to the plasma membrane and its mobilization to dynamic ruffling protrusions at the cell front.
These RCP-driven changes in $\alpha 5 \beta 1$ integrin trafficking led to a marked increase in fibronectin-dependent migration of tumour cells into 3D matrices.

Recycling of $\alpha 5 \beta 1$ integrin had no effect on ligand binding and adhesive function of $\alpha 5 \beta 1$ integrin, which prompted the authors to examine whether $\alpha 5 \beta 1$ integrin recycling affects receptor Tyr-kinase signalling. Indeed, RCP was required for the interaction between $\alpha 5 \beta 1$ integrin and epidermal growth factor receptor-1 (EGFR1): knockdown of $\alpha 5 \beta 1$ integrin inhibited the coprecipitation of EGFR1with RCP, and knockdown of RCP inhibited EGFR1 recycling. So, recruitment of RCP to $\alpha 5 \beta 1$ integrin is required for subsequent association of RCP with EGFR1 and enhanced EGFR1 recycling (this was associated with increased autophosphorylation of the receptor and activation of the pro-invasive kinase AKT/protein kinase B). The authors propose that RCP provides a scaffold that promotes the physical association and coordinated trafficking of $\alpha 5 \beta 1$ integrin and EGFR1, and that this drives the migration of tumour cells into 3D matrices.

Ekat Kritikou

ORIGINAL RESEARCH PAPERS Caswell, P. T.

et al. Rab-coupling protein coordinates recycling of $\alpha 5 \beta 1$ integrin and EGFR1 to promote cell migration in 3D microenvironments. J. Cell Biol. 6 Oct 2008 (doi:10.1083/jcb200804140)| Pellinen, T. et al. Integrin trafficking regulated by Rab21 is necessary for cytokinesis. Dev. Cell 15, 371-385 (2008) 